\title{
Approach for the Design of a Broadband Microwave Power Amplifier in Microstrip Technology for Mobile Communications Systems
}

\author{
Lahsaini Mohammed $^{1}$, Zenkouar Lahbib ${ }^{2}$, Saadi Adil ${ }^{3}$ \\ ${ }^{1}$ Electronics, Communication Systems and Energy Optimization Group, Faculty of Sciences, Moulay Ismail University, Meknès, \\ Morocco \\ ${ }^{2}$ Equipe de Recherche en Smart Communications (ERSC), E3S Research Center, Mohammadia School of Engineers (EMI), \\ Mohammed $\mathrm{V}^{\text {th }}$ University, Rabat, Morocco \\ ${ }^{3}$ Control and supervision of systems, Ecole Nationale Supérieure d'Arts et Métiers, Moulay Ismail University, Meknès, Morocco
}

\begin{tabular}{l} 
Article Info \\
\hline Article historys: \\
Received Oct 12, 2019 \\
Revised May 18, 2020 \\
Accepted Jun 5, 2020 \\
\hline
\end{tabular}

\section{Keywords:}

Microwaves

Broadband power amplifier

Microstrip bias circuit

Impedance matching

Quarter wave transformer

Linearity

\begin{abstract}
This work presents a broadband power amplifier in S-band in microstrip technology. The proposed power amplifier is modeled with a single-stage architecture based on a field effect transistor ATF13786 of Agilent Technologies (hp $)^{\circledR}$. The used transistor has been biased with transmission lines and it has been matched with networks in the form of $\lambda / 4$ transformers filters at the input and at the output. This amplifier has been studied and optimized using the Advanced Design System (ADS $\left.{ }^{\circledR}\right)$ software. The simulation results of the output power and $S$ parameters show excellent characteristics with a satisfactory gain greater than $10.9 \mathrm{~dB}$, low reflections, a saturated output power of $16.4 \mathrm{dBm}$ with a $1 \mathrm{~dB}$ compression point at an input power level of about $5 \mathrm{dBm}$, a maximum PAE of $25.3 \%$ and unconditional stability in the desired frequency band. The modeled amplifier can be integrated into mobile communications systems namely LTE mobile networks (2500 to $2690 \mathrm{MHz}$ ) and wireless networks using Wi-Fi protocol (2400 to $2485 \mathrm{MHz}$ ).
\end{abstract}

Copyright $\odot 2019$ Institute of Advanced Engineering and Science. All rights reserved.

\section{Corresponding Author:}

Lahsaini Mohammed,

Materials and Instrumentation Group, LASMAR,

Faculty of Science, Moulay Ismail University,

Meknes, Morocco

Email: mohammed.lahsaini@gmail.com

\section{INTRODUCTION}

The design of power amplifiers is today the subject of particular attention in the world of microwaves. On the one hand, the current development of communications systems is such that it requires occupying ever-higher frequency bands. On the other hand, within the sub-systems of transmission-reception, amplifiers occupy a preponderant place because they condition, in general, the quality of transmission of information. The design of a broadband power amplifier requires accurate modeling of the RF active device, effective impedance matching, stability throughout operation and ease of practical implementation [1-3].

The characteristics of a wideband power amplifier are evaluated by its ability to achieve maximum power gain over the required frequency bandwidth, under stable operating conditions with a minimum of amplification stages and the need for high efficiency or linearity can be taken into account if necessary. We note that, for stable operation, it is necessary to evaluate the operating frequency domains in which the active device may be potentially unstable $[4,5]$.

A single-stage power amplifier can be modeled by the circuit of figure 1, where the matching networks are used on both sides of the transistor to transform the input/output impedance $Z_{0}$ in source impedance $Z_{s}$ and load impedance $\mathrm{Z}_{\mathrm{L}}$ [6-8]. 


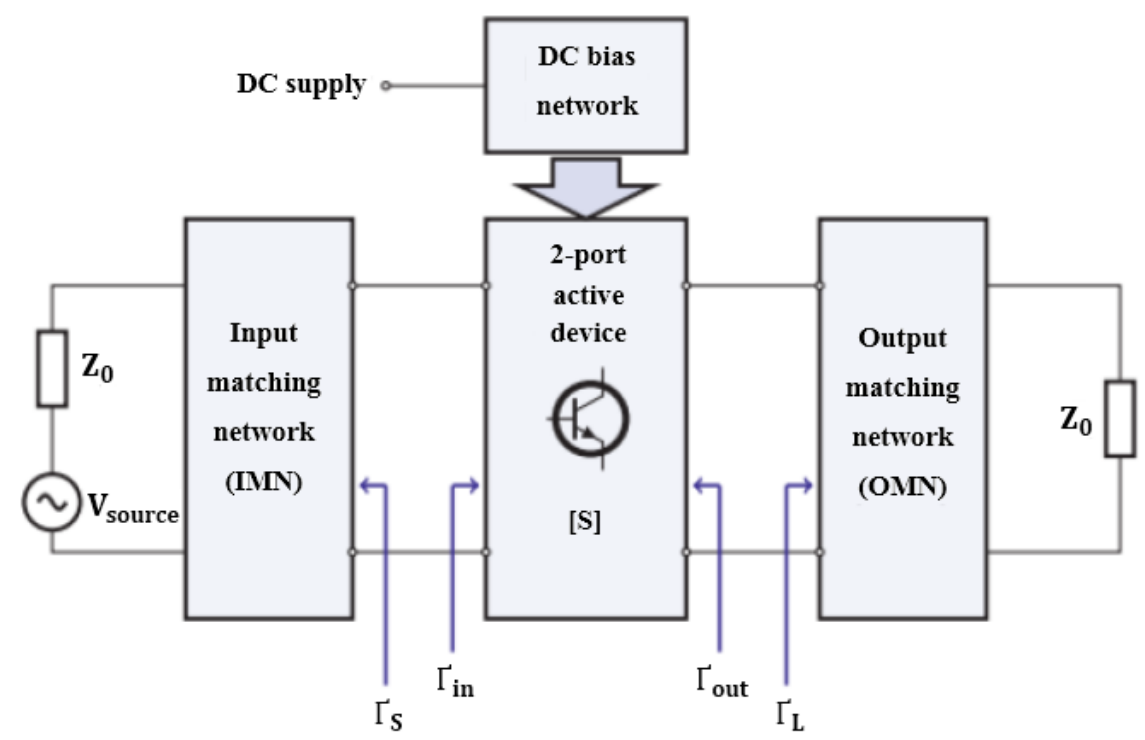

Figure 1. Block diagram of a microwave amplifier [5]

Our work is a contribution to the design of microwave circuits in microstrip technology in order to make amplifiers operating in linear and non-linear modes. We present, in this article, a design methodology, its mathematical formalism as well as the optimization procedure associated with it. Then the study, modeling and characterization of an amplifier for S-band applications are detailed.

The proposed amplifier has high and significant output power, broadband impedance matching, and minimal reflection losses with satisfactory power gain.

\section{BIASING NETWORKS}

Biasing networks are important elements for the design of amplifiers. They provide the means of efficient excitation for the transistors. The biasing parameters may vary with the technology of the transistors; however, the design principles for the biasing methods are basically the same. The objective of this axis is to include a complete treatment of biasing techniques used for UHF frequencies to millimeter waves [6-10].

\subsection{Biasing of transistors}

The biasing of transistors comprises two parts: the selection of biasing Q-point for optimum performance of the device in terms of gain, noise figure, output power, PAE and linearity, and the biasing networks. Both are essential to the operation of amplifiers, especially regarding the low levels of noise and high power $[5,6]$.

Basically, a biasing network is composed of a DC block and RF inductance, The RF inductance aims to have a very high impedance at the operating frequency to prevent RF from leaking through the biasing network. In this case, the effect of the biasing network is negligible when it has a very high impedance for matching networks. Chip capacitors in hybrid circuits and MIM capacitors in MMICs are generally used as DC bias blocks. Ideally, the capacitor should have a short circuit at operating frequencies. The values of the capacities must be selected so that $\omega C=2 \pi f C$ be very large. Therefore, $Z_{C}=-j(1 / \omega C)$ is very small and the signal will pass through the capacitor with little loss or reflection [6].

\subsection{Microstrip biasing circuit}

The RF choke at microwave frequencies is generally realized using a high-impedance $\lambda / 4$ line, also known as a parallel stub terminated by a bypass capacitor $C_{B}$, as shown in figure $2 \mathrm{a}$ [6]. A DC block may be a capacitor or a back wave coupler $3 \mathrm{~dB}$ described in reference [6]. For low RF leakage across the biasing network, the impedance ratio of the stub in parallel $\left(Z_{S}\right)$ and the line impedance $\left(Z_{0}\right)$ through, $Z_{S} / Z_{0}$, The low-high impedance lines serve as a lowpass filter, which prevents the microwave signal leaking into the bias port. In this case, the bandwidth increases when the impedance of the stub increases. For VSWR $\leq 1.05$, the bandwidth for $Z_{S}=100 \Omega$ is about $12 \%$. In order to further increase the bandwidth, two sections of quarter-wave transmission lines are used. If an open circuit is required across the main line for RF signals, a quarter-wave high-impedance line followed by an open circuited quarter-wave low-impedance line are connected. The configuration is illustrated in figure $2 b$ [6]. Assuming that the characteristic impedance $Z_{0}$ of the through line is the same as the impedance of the system, the normalized admittance with the load $\left(Y_{0}=1 / Z_{0}\right)$ is 
With

$$
y=\frac{Y}{Y_{0}}=1+\frac{Z_{0}}{Z_{\text {in }}}
$$

$$
Z_{\text {in }}=j Z_{1} \frac{Z_{1} \tan \theta_{1} \tan \theta_{2}-Z_{2}}{Z_{1} \tan \theta_{2}+Z_{2} \tan \theta_{1}}
$$

Here, $\theta_{1}, Z_{1}$ and $\theta_{2}, Z_{2}$ are respectively the electrical length of the line, the characteristic impedance of the first and second line sections. Under matched conditions, $Z_{\text {in }}=\infty$ and $y=1$. The maximum bandwidth is obtained when $Z_{1} / Z_{2}$ is wide. For example, with $Z_{1}=100 \Omega, Z_{2}=10 \Omega, Z_{0}=50 \Omega$, and VSWR $=1.2$, the maximum bandwidth is about $40 \%$. Generally, the low impedance line section is replaced by a radial line section, as shown in Figure $2 c[6,11]$. This arrangement provides better bandwidth than a $\lambda / 4$ open circuited line section and is smaller in size. The transmission line medium could be either strip line or microstrip. In this biasing scheme, the biasing circuitry becomes an integral part of the amplifier design [6].

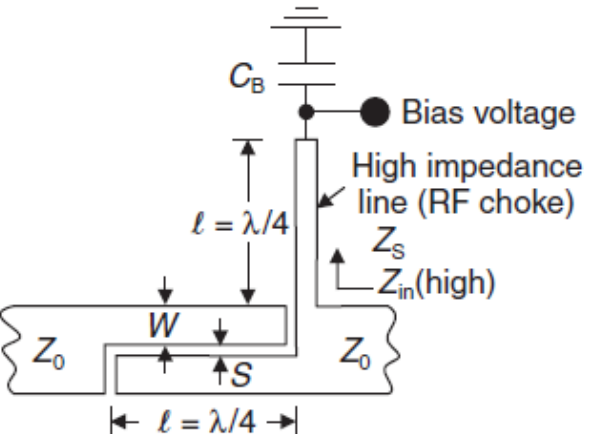

(a)

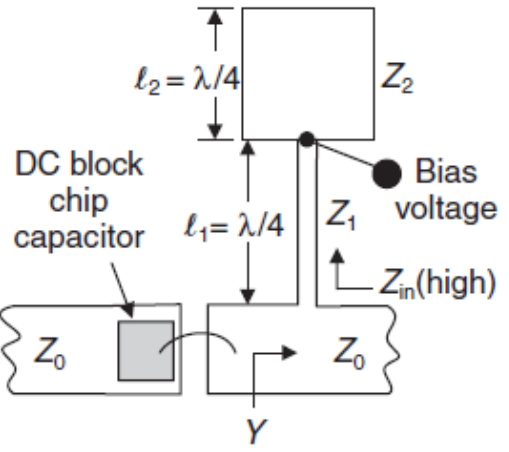

(b)

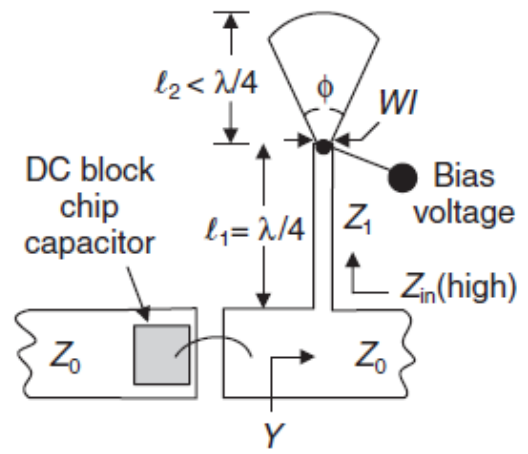

(c)

Figure 2. Simplified microwave biasing circuits: (a) coupled-line DC block and $\lambda / 4$ transformer, (b) MIM capacitor DC block and two $\lambda / 4$ transformers, and (c) MIM capacitor DC block and a $\lambda / 4$ transformer in series with a radial line stub [6].

\section{IMPEDANCE MATCHING}

Impedance matching plays an important role in the design of microwave circuits. The design of a microwave amplifier with inadequate impedance matching will influence the stability of the circuit and reduce its efficiency. In fact, the use of matched devices is related to several objectives. The most important factor is the maximum power transfer.

One way to match one or more circuits is to insert a quarter-wave line between the impedance of the load and the input impedance line and to choose a suitable characteristic impedance. This is one of the simplest techniques to perform impedance matching in a narrow band $[9,11]$.

Impedance transformers generally include uniform quarter-wave line sections in cascade. Discontinuities result from different impedance jumps, such as a change in the width of the microstrip line. Upon selection of the matching network, certain factors must be taken into account, such as the complexity, the bandwidth and the necessary adjustment according to the desired application.

The relation which links the characteristic impedance $\mathrm{Z}_{0}$ and the load impedance $\mathrm{Z}_{\mathrm{L}}$ to the impedances characterizing the multi-section transformer is calculated from the following formula [12]:

$$
\ln \frac{\mathrm{z}_{\mathrm{n}+1}}{\mathrm{Z}_{\mathrm{n}}}=2^{-\mathrm{N}} \mathrm{C}_{\mathrm{n}}^{\mathrm{N}} \ln \frac{\mathrm{Z}_{\mathrm{L}}}{\mathrm{z}_{0}}
$$

To achieve matching, we will study the case of three sections of length $(\lambda / 4)$, as shown in figure 3 :

The equivalent characteristic impedances obtained by applying the equation (3) are given by:

$$
\begin{aligned}
& \mathrm{Z}_{1}=\sqrt[8]{\mathrm{Z}_{\mathrm{L}} \mathrm{Z}_{0}^{7}} \\
& \mathrm{Z}_{2}=\sqrt{\mathrm{Z}_{0} \mathrm{Z}_{\mathrm{L}}} \\
& \mathrm{Z}_{3}=\sqrt[8]{\mathrm{Z}_{0} \mathrm{Z}_{\mathrm{L}}^{7}}
\end{aligned}
$$




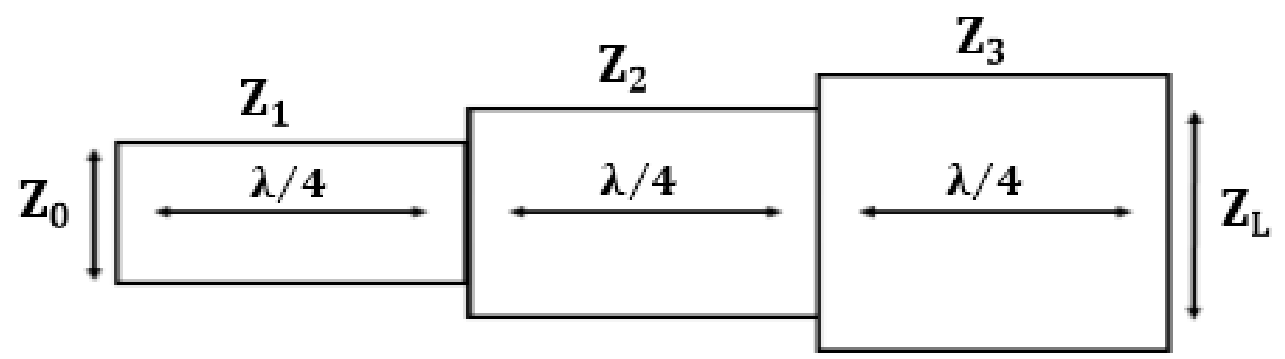

Figure 3. A three sections quarter-wave transformer [12]

\section{STABILITY}

A power amplifier must not oscillate whatever the impedance presented to it, both at the input and at the output. The stability criteria make it possible to rule on the risks of instability of the power amplifier. So the combination of the condition of Rollet [15-21]:

$$
\mathrm{K}=\frac{1-\left|\mathrm{S}_{11}\right|^{2}-\left|\mathrm{S}_{22}\right|^{2}+|\Delta|^{2}}{2\left|\mathrm{~S}_{12} \mathrm{~S}_{21}\right|}>1
$$

With one of the following conditions:

$$
\begin{gathered}
B_{1}=1+\left|S_{11}\right|^{2}-\left|S_{22}\right|^{2}-|\Delta|^{2}>0 \\
B_{2}=1-\left|S_{11}\right|^{2}+\left|S_{22}\right|^{2}-|\Delta|^{2}>0 \\
|\triangle|=\left|S_{11} S_{22}-S_{12} S_{21}\right|<1 \\
1-\left|S_{11}\right|^{2}>\left|S_{12} S_{21}\right| \\
1-\left|S_{22}\right|^{2}>\left|S_{12} S_{21}\right|
\end{gathered}
$$

provide unconditional stability over the entire of the considered frequency band [15].

The $\boldsymbol{S}_{\boldsymbol{i j}}$ parameters are the scattering parameters of the transistor or the amplifier.

A new and unique stability criterion has been developed recently [17, 21]. The condition:

$$
\mu_{1}=\frac{1-\left|S_{11}\right|^{2}}{\left|S_{22}-S_{11}{ }^{*} \Delta\right|+\left|S_{12} S_{21}\right|}>1
$$

Or :

$$
\mu_{2}=\frac{1-\left|S_{22}\right|^{2}}{\left|S_{11}-S_{22}{ }^{*} \Delta\right|+\left|S_{12} S_{21}\right|}>1
$$

alone ensures unconditional stability.

\section{PROPOSED BROADBAND POWER AMPLIFIER}

Using the concepts mentioned above, the proposed amplifier is shown in Figure 4. For the design of our broadband power amplifier we used the commercialized transistor ATF13786 of Agilent Technologies (hp) ${ }^{\circledR}$, which is a field effect transistor.

In the design process, the biasing condition (known as DC Q-point) for the active device must be carefully selected because it determines the basic performance in terms of stability, output power, gain and PAE of the entire power amplifier. The biasing network used is shown in figure 4. The proposed power amplifier is biased with two voltage sources $\mathrm{V}_{\mathrm{DS}}$ of $+2,8 \mathrm{~V}$ and $\mathrm{V}_{\mathrm{GS}}$ of $-0,5 \mathrm{~V}$. As necessary for bandwidth, the RF choke is realised by using a high impedance transmission line of length $\lambda / 4$ followed by a radial stub $[6,11]$. This combination helps prevent RF energy from entering the power supply and increases bandwidth. 


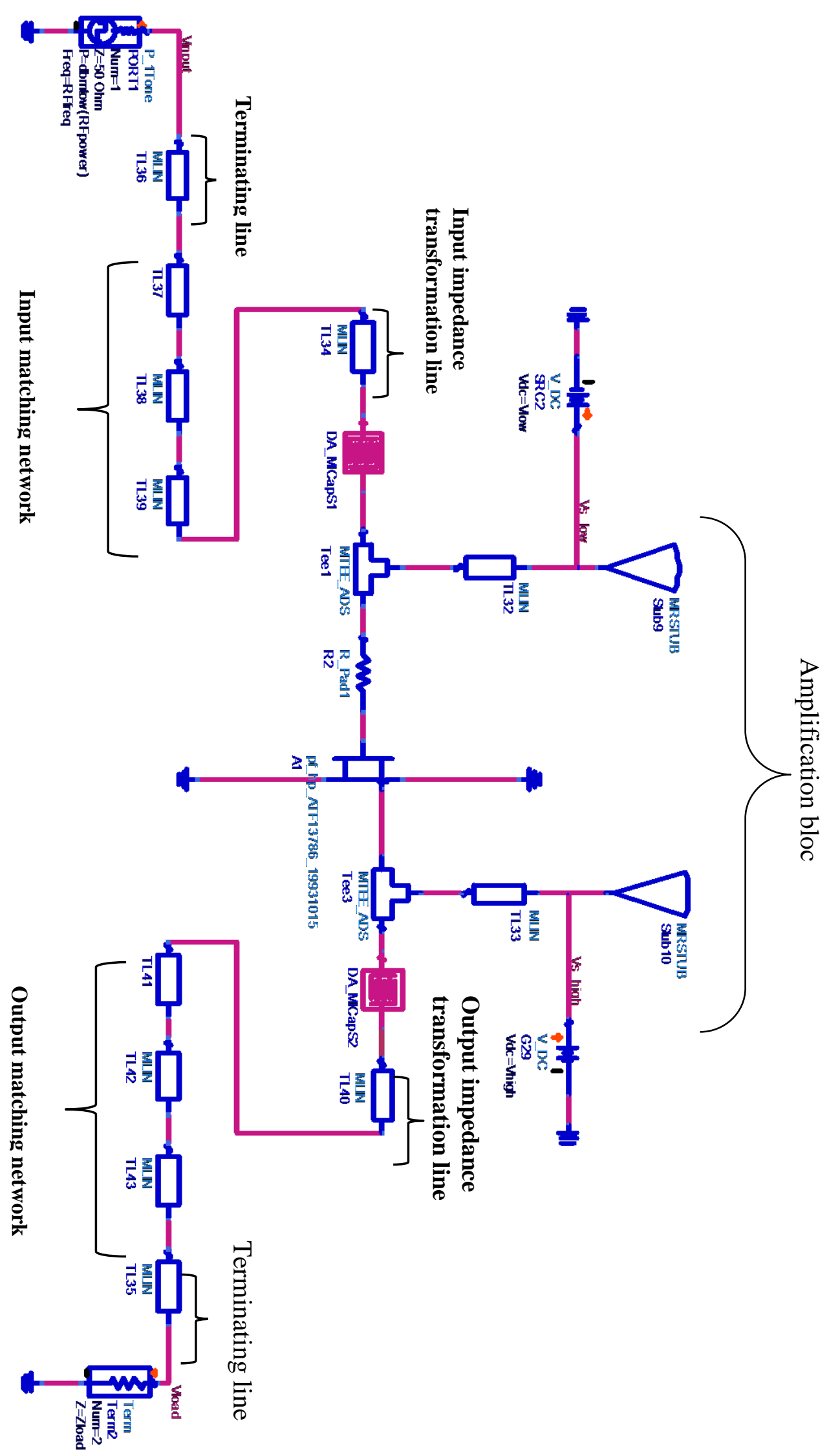

Figure 4. Schematic of the proposed single-stage broadband power amplifier 
The DC Block was realized with a Microstrip Interdigital Capacitor (DA_MICapS1 and DA_MICapS2) in order to avoid the use of the localized elements to facilitate the fabrication of the circuit and to reduce the noise and the energy losses.

As shown in figures 4 and 5, the input and output matching networks were designed as simple as possible to reduce both the consumption of the layout and loss of gain. The design of the two matching networks depends on the frequency band in which this broadband power amplifier is to be designed.

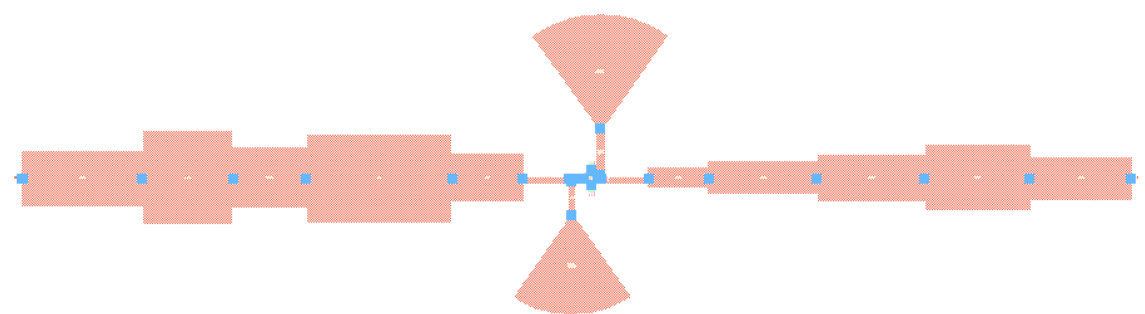

Figure 5. Layout of the proposed single-stage broadband power amplifier

In this work, the proposed amplifier is implemented on a substrate of type FR4 epoxy, with a permittivity $(\varepsilon r)$ equal to 4.32 and a thickness equal to $\mathrm{h}=1.6 \mathrm{~mm}$.

\section{RESULTS AND DISCUSSION}

The complete amplifier circuit was simulated using the ADS software of Keysight ${ }^{\circledR}$. Figures 6 to 11 present the results of these simulations which will constitute the reference data for the experimental characterization.

Figure 6 shows us; on the frequency band [2-3] GHz, the losses by reflection in input $\left(\mathrm{S}_{11}\right)$ and in output $\left(\mathrm{S}_{22}\right)$ for low levels of excitation. From figure 6, we can observe that the input reflection losses are less than $-10.647 \mathrm{~dB}$ and can reach the value $-36.523 \mathrm{~dB}$ at $2.88 \mathrm{GHz}$. On the other hand, at the output, these losses are less than $-11.455 \mathrm{~dB}$ and can reach the value $-44.869 \mathrm{~dB}$ at $2.73 \mathrm{GHz}$.

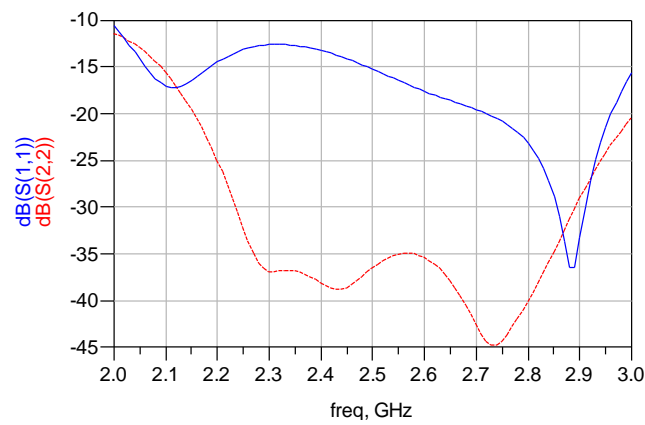

Figure 6. Reflexion coefficients $\mathrm{S}_{11}$ and $\mathrm{S}_{22}$

The simulated gain $\mathrm{S}_{21}$ for the proposed BPA is given in figure 7. As can be seen, the small signal gain varies between a minimum value of $10.944 \mathrm{~dB}$ at $3 \mathrm{GHz}$ and a maximum value of $13.979 \mathrm{~dB}$ at $2.07 \mathrm{GHz}$. Inverse isolation $\mathrm{S}_{12}$ represents the internal return of the output to the input of a two-port device. For simulated inverse isolation of the proposed BPA. As can be seen in figure $7, \mathrm{~S}_{12}$ varies between a minimum value of $-25.767 \mathrm{~dB}$ at $2 \mathrm{GHz}$ and a maximum value of $-23.889 \mathrm{~dB}$ at $2.95 \mathrm{GHz}$.

In terms of practical operation of a power amplifier, it is necessary that $S_{12}$ be as small as possible because the lower reverse isolation value is the higher degree of isolation between the output and the input, as well as the higher degree of stability of a given stage.

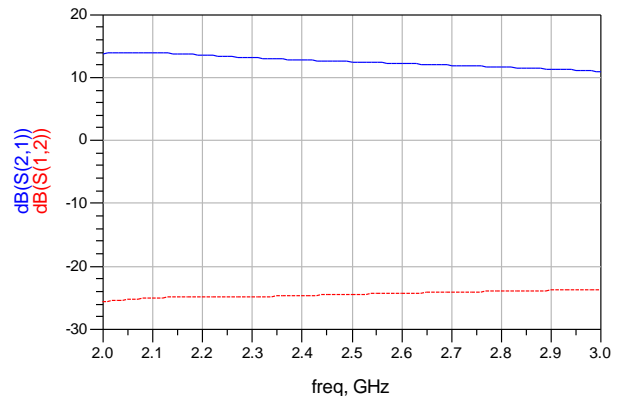

Figure 7. Transmission coefficients $\mathbf{S}_{\mathbf{2 1}}$ and $\mathbf{S}_{\mathbf{1 2}}$ 
Figure 8 shows the values of the stability coefficients $\mathrm{k}$ (StabFact1) and $\mu$ (Muprime1 and Mu1) simulated on the band [2-3] GHz. The stability factors are greater than 1 over the entire operating band, the unconditional stability conditions of the proposed BPA are met. This means that any source or load can be connected to the input or the output of BPA without risk of becoming unstable or producing oscillations.

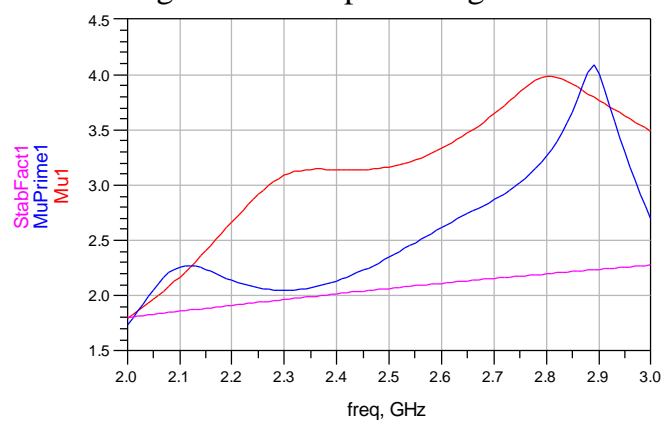

Figure 8. Stability factors

The $1 \mathrm{~dB}$ compression point is defined as the point at which the output power level corresponds to a $1 \mathrm{~dB}$ difference from the ideal linear behavior. The corresponding input power level is used to mark the boundary between linear and nonlinear regions.

Figures 9 and 10 show the simulated output power and the PAE of the proposed BPA. The curves were plotted at the central operating frequency equal to $2.5 \mathrm{GHz}$. For each of them, the results are presented according to the input power $\mathrm{P}_{\mathrm{e}}$ of the circuit.

Figure 9 shows that the simulated output power reaches a maximum of $16.403 \mathrm{dBm}$ for $20 \mathrm{dBm}$ at the input power, resulting in an output power of $43.682 \mathrm{~mW}$. The proposed BPA achieves a $1 \mathrm{~dB}$ compression at an input power level of about $5 \mathrm{dBm}$. As shown in figure 10 , at $2.5 \mathrm{GHz}$, the simulated PAE reaches a maximum value of $25.317 \%$ for an input power of $6 \mathrm{dBm}$.

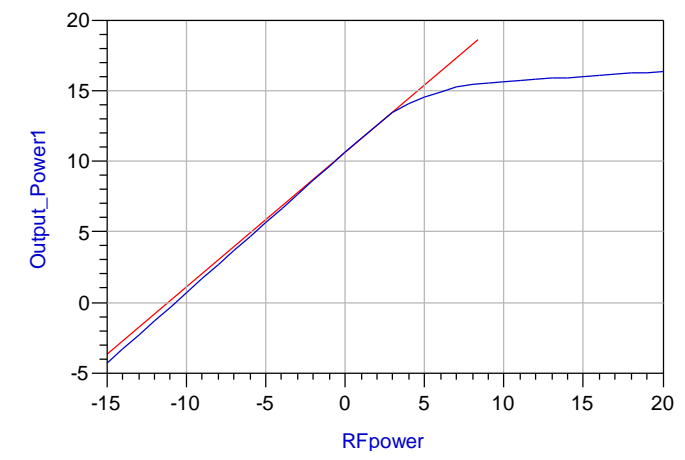

Figure 9. Output power versus the input power

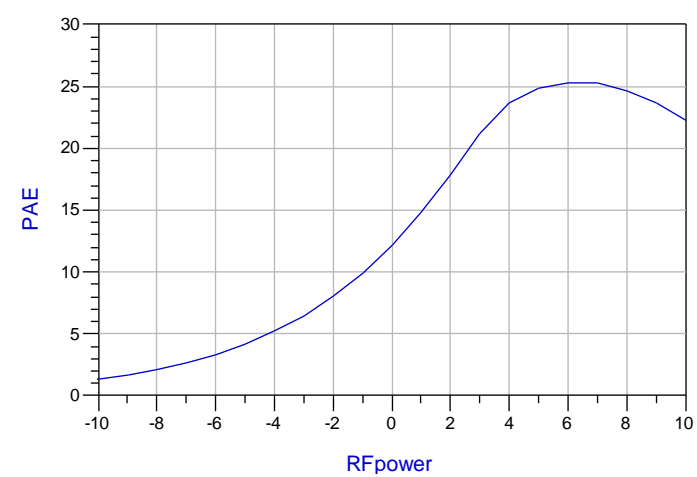

Figure 10. Added power efficiency versus the input power

Figure 11 shows the curve of the noise figure as a function of frequency. The simulated noise figure $\mathrm{F} \_\mathrm{dB}$ varies between 4.386 and $6.168 \mathrm{~dB}$ over the frequency band [2-3] GHz. The lowest NFmin value is $1.847 \mathrm{~dB}$ obtained at $2 \mathrm{GHz}$, as shown in figure 11 . 


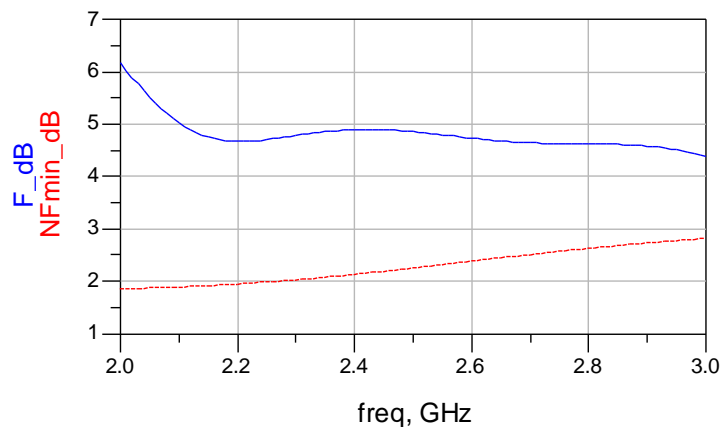

Figure 11. Noise figure

\section{COMPARISON TABLE}

In the table 1, the performance of the designed broadband amplifier is summarized and compared to the state of art of broadband power amplifiers operating in the $\mathrm{S}$ band.

Comparing current work with state-of-art of the BPA [22-24], we can clearly see that the proposed broadband matching techniques are useful, and the designed BPA achieves excellent bandwidth with good matching on all frequency bands of operation. The simulated reflection loss shows good results with a bandwidth of $1 \mathrm{GHz}$ compared to articles [22-24].

In the frequency range from $2 \mathrm{GHz}$ to $3 \mathrm{GHz}$, the proposed BPA achieves a maximum gain of $13.979 \mathrm{~dB}$, and a $\mathrm{P}_{\mathrm{sat}}$ of $16.403 \mathrm{dBm}$ with a $\mathrm{PAE}_{\max }$ of $25.317 \%$ and a $\mathrm{P}_{1 \mathrm{~dB}}$ of approximately $5 \mathrm{dBm}$ under power supply voltages of $+2.8 \mathrm{~V}$ for $\mathrm{V}_{\mathrm{ds}}$ and $-0.5 \mathrm{~V}$ for $\mathrm{V}_{\mathrm{gs}}$.

Given broadband input power and broadband frequency coverage, the proposed BPA offers better broadband performance than other BPAs [22-24], and has high gain, excellent bandwidth, good output power and simple circuits. It is a good candidate for wideband PAs operating in the $\mathrm{S}$ band.

Table 1. State of art of broadband power amplifiers

\begin{tabular}{cccccccccc}
\hline \hline Ref. & $\begin{array}{c}\text { Freq. } \\
(\mathrm{GHz})\end{array}$ & $\begin{array}{c}\text { Gain } \\
(\mathrm{dB})\end{array}$ & $\begin{array}{c}\mathrm{S}(1,1) \\
(\mathrm{dB})\end{array}$ & $\begin{array}{c}\mathrm{S}(2,2) \\
(\mathrm{dB})\end{array}$ & $\begin{array}{c}\mathrm{S}(1,2) \\
(\mathrm{dB})\end{array}$ & $\begin{array}{c}\text { Psat } \\
(\mathrm{dBm})\end{array}$ & PAE (\%) & $\begin{array}{c}\text { NF } \\
(\mathrm{dB})\end{array}$ & $\begin{array}{c}\text { VDS } \\
(\mathrm{V})\end{array}$ \\
\hline [This work] & {$[2-3]$} & 13,9 & $\begin{array}{c}-10.6 / \\
-36,5\end{array}$ & $\begin{array}{c}-11,4 / \\
-44,8\end{array}$ & $\begin{array}{c}-23,8 / \\
-25,7\end{array}$ & 16,4 & 25,3 & $<6,1$ & 2.8 \\
{$[22]$} & {$[1,9-2,7]$} & 11 & - & - & - & 28.1 & 13.7 & - & 2.5 \\
{$[23]$} & {$[1,8-2,8]$} & 25 & $-12 /$ & $-9 /$ & - & 28 & 6.1 & - & 5 \\
{$[24]$} & {$[1,75-2,15]$} & $10-11,7$ & $-11 /$ & $-13 /$ & $-18 /$ & 8 & - & - & 12 \\
\hline \hline
\end{tabular}

\section{CONCLUSION}

In conclusion, the design and analysis of a microwave power amplifier in microstrip technology was presented. The simulation results show that the proposed amplifier offers excellent performance for the applications of LTE mobile networks and wireless networks using Wi-Fi protocol. The proposed design has a power gain of $13.979 \mathrm{~dB}$ with reverse isolation below $-23.8 \mathrm{~dB}$ under a power supply of $2.8 \mathrm{~V}$ for $\mathrm{V}_{\mathrm{ds}}$ and $\quad-0.5 \mathrm{~V}$ for $\mathrm{V}_{\mathrm{gs}}$. The proposed power amplifier has successfully achieved good performance in terms of stability and impedance matching. This amplifier is capable of delivering an output power of up to $16.403 \mathrm{dBm}$.

\section{REFERENCES}

[1] G. Rafael-Valdivia and O. Castellanos Ballesteros, "New methodology for modeling, design and implementation of RF power amplifiers," Journal of Microwaves, Optoelectronics and Electromagnetic Applications, vol. 16, no. 3, pp. 785-800, September 2017.

[2] M. Hazouard, "Conception et réalisation d'amplificateurs micro-ondes de puissance à l'aide de la méthode des fréquences réelles," Université Bordeaux I, 2002.

[3] G. Gonsalez, Microwave Transistor Amplifiers, Analysis and Design, 2 ed., Prentice Hall,, August 30, 1996.

[4] A. Rachakh, L. El Abdellaoui , J. Zbitou, A. Errkik, A. Tajmouati and M. Latrach, "A New Design of a Microstrip Microwave Broadband Power Amplifier for GSM, DCS and PCS Bands," International Journal of Microwave and Optical 
Technology, vol. 13, no. 6, pp. 493-500, 2018.

[5] M. Ribate, J. Zbitou, R. Mandy, A. Erkik and M. Latrach, "Broadband GaAs FET Power Amplifier for L and S Bands Applications," International Journal of Intelligent Engineering and Systems ,vol. 11, no. 5, pp. 96-104, April 2018.

[6] Inder J. Bahl, Fundamentals of RF and Microwave Transistor Amplifiers, John Wiley \& Sons, 2009.

[7] D. K. Misra, Radio-Frequency and Microwave Communication Circuits; Analysis and Design, Wiley-Blackwell, John Wiley \& Sons, Inc., Hoboken, New Jersey, 2004.

[8] B. Razavi, RF Microelectronics, 2 ed., P. Hall, Ed, 2011.

[9] R. Mongia, I. Bahl, P. Bhartia, and J. Hong, RF and Microwave Coupled-Line Circuits, 2 ed., Artech House, Norwood, 2007.

[10] I. J. Bahl and P. Bhartia, Microwave Solid State Circuit Design, 2 ed., John Wiley \&Sons, Hoboken, NJ, 2003.

[11] R. Feghhi and M. Joodaki, "Realization of a broadband hybrid X-band power amplifier based on fT-doubler technique," International Journal of Electronics and Communications (AË̈), vol. 104, pp. 119-127, 2019.

[12] I. Toulali, M. Lahsaini and L. Zenkouar, "Design of a Low Noise Amplifier Using the Quarter Wave Transformers Matching Technique in the Frequency Band [9-13] GHz," International Journal on Communications Antenna and Propagation, vol. 5, no. 4, pp. 248-255, 2015.

[13] R. E. Collin, Foundations for Microwave Engineering, 2 ed., New York: John Wiley \& Sons, 2001.

[14] D. M. Pozar, Microwave engineering, 3 ed., New York: John-Wiley \& Sons, 2005.

[15] M. \&. S. J. Edwards, "A new criterion for linear 2-port stability using a single geometrically derived parameter," IEEE Transactions on Microwave Theory and Techniques, vol. 40, no. 12, pp. $2303-2311,1992$.

[16] A. Peiman , "Design and implementation of a high efficiency RF power amplifier for S-band telemetry subsystems," International Journal of Electronics and Communications (AEÜ), vol. 70, no. 9, pp. 1311-1320, 2016.

[17] G. \&. N. B. Lombardi, "Criteria for the evaluation of unconditional stability of microwave linear two-ports: a critical review and new proof," IEEE Transactions on Microwave Theory and Techniques, vol. 47, no. 6, pp. 746 - 751, 1999.

[18] A. Mohsen, M. Jaafar Ayoub, M. Alloush, A. Harb, N. Deltimple and A. Serhane, "Common source power amplifier design for 5G application in 28-nm UTBB FD-SOI technology," International Journal of Electronics and Communications (AË̈), vol. 96, pp. 273-278, November 2018.

[19] Tan, E. L., Sun, X., \& An, K. S., "Unconditional stability criteria for microwave networks," PIERS Proceedings, pp. 15241528, Beijing, China, March 23-27,2009.

[20] M. K. Salama and A. M. Soliman, "Low-voltage low-power CMOSRF low noise amplifier," International Journal of Electronics and Communications (AË̈), vol. 63, no. 6, pp. 478-482, June 2009.

[21] M. Lahsaini, L. Zenkouar and S. Bri , "Modeling of a Microwave Amplifier Operating around $11 \mathrm{GHz}$ for Radar Applications," International Journal of Electrical and Computer Engineering (IJECE), vol. 8, no. 5, pp. 3496-3503, 2018.

[22] B. Francois and P. Reynaert, "Highly linear fully integrated wideband RF PA for LTE-advanced in 180-nm SOI," IEEE Transactions on Microwave Theory and Techniques, vol. 63, no. 3, p. 649-658, 2015.

[23] C.Q. Chen, M.L. Hao, Z.Q. Li, Z.B. Du, and H. Yang, "A 1.8-2.8 GHz Highly Linear Broadband Power Amplifier for LTE-A Application," Progress in Electromagnetics Research C, vol. 66, p. 47-54, 2016.

[24] A. Rachakh, L. El Abdellaoui, J. Zbitou, A. Errkik, A. Tajouati, and M. Latrach, "A Novel Configuration of a Microstrip Microwave Wideband Power Amplifier for Wireless Application," Telkomnika, vol. 16, no. 1, pp. 2014-2031, 2018. 